\title{
Relationship between surgeon volume and outcomes in spine surgery: a dose-response meta-analysis
}

\author{
Hui-Zi Li ${ }^{1,2 \#}$, Zhong Lin ${ }^{2,3 \#}$, Zong-Ze Li ${ }^{4 \#}$, Zeng-Yan Yang ${ }^{4}$, Yang Zheng ${ }^{5}$, Yong Li $^{4}$, Hua-Ding Lu ${ }^{1,2}$ \\ ${ }^{1}$ Department of Orthopaedics, The Fifth Affiliated Hospital of Sun Yat-sen University, Zhuhai 519000, China; ${ }^{2}$ Guangdong Provincial Engineering \\ Research Center of Molecular Imaging, ${ }^{3}$ Center for Interventional Medicine, the Fifth Affiliated Hospital, Sun Yat-sen University, Zhuhai 519000, \\ China; ${ }^{4}$ Department of Orthopaedics, The People's Hospital of Ruijin City, Ruijin 342500, China; Department of General Surgery, the Fourth \\ Affiliated Hospital of Nanchang University, Nanchang 330006, China \\ Contributions: (I) Conception and design: HZ Li, Z Lin, HD Lu, ZZ Li, ZY Yang; (II) Administrative support: None; (III) Provision of study materials \\ or patients: None; (IV) Collection and assembly of data: All authors; (V) Data analysis and interpretation: All authors; (VI) Manuscript writing: All \\ authors; (VII) Final approval of manuscript: All authors. \\ \#These authors contributed equally to this work. \\ Correspondence to: Hua-Ding Lu. Department of Orthopedics, the Fifth Affiliated Hospital of Sun Yat-sen University, Zhuhai 519000 , China. \\ Email: johnniehuading@163.com.
}

Background: The relationship between surgeon volume and outcomes in spine surgery is unclear and published studies report inconsistent results. Therefore, a dose-response meta-analysis was conducted to clarify the influence of surgeon volume on outcomes in spine surgery.

Methods: PubMed, Embase, and The Cochrane Library were systematically searched without language limitation for observational studies which investigated the relationship between surgeon volume and outcomes in spine surgery. The primary outcome was postoperative morbidity and the secondary outcomes consisted of mortality, length of hospital stay, readmission, and hospital costs. For binary variable and continuous variable, odds ratios (ORs) with 95\% CIs and weighted mean differences (WMDs) with 95\% CIs were pooled respectively. Additionally, a dose-response meta-analysis was performed for the primary outcome.

Results: Eleven studies with 1,986,545 patients were included in the current meta-analysis. Pooled estimate indicated that a higher surgeon volume was associated with lower postoperative morbidity (OR, 0.62; 95\% CI: $0.52-0.75 ; \mathrm{I}^{2}=93.9 \%$ ), lower mortality (OR, 0.76; 95\% CI: $0.66-0.87 ; \mathrm{I}^{2}=0$ ), shorter length of hospital stay (WMD, -7.07 ; 95\% CI: -7.08 to -7.06 ; $\mathrm{I}^{2}=100 \%$ ), less readmission (OR, 0.78; 95\% CI: $0.72-$ $0.85 ; \mathrm{I}^{2}=93.1 \%$ ), and lower hospital costs (WMD, $-25,497.47 ; 95 \%$ CI: $-25,528.43$ to $\left.-25,466.51 ; \mathrm{I}^{2}=100 \%\right)$.

Dose-response analysis suggested a nonlinear relationship between surgeon volume and postoperative morbidity (P for nonlinearity less than 0.00001).

Conclusions: The current evidence indicate that higher surgeon volume is associated with lower morbidity and mortality, shorter length of hospital stay, less readmission, and lower hospital costs in spine surgery.

Keywords: Surgeon volume; treatment outcome; spine surgery; meta-analysis

Submitted Apr 16, 2018. Accepted for publication Sep 21, 2018.

doi: $10.21037 /$ atm.2018.10.48

View this article at: http://dx.doi.org/10.21037/atm.2018.10.48 


\section{Introduction}

Irrespective of a continuous economic downturn in the United States, the cumulative number of spine surgeries and individual patient's hospital costs increase significantly since $2001(1,2)$. In spite of the advances in surgical procedures and perioperative management during these years, spine surgery as a high-risk complicated surgical procedure is still associated with high morbidity, mortality, readmission, and hospital costs and impose a huge burden on the national health system (3-5). Theoretically, abundant surgical experience should translate into better outcomes (6-8). The number of operations which a surgeon performed annually (i.e., surgeon case volume) is an indirect reflection for surgical experience (9). In fact, the volume-outcomes relationship has been evaluated in some surgical conditions, since Hannan et al. reported it in 1989 (10-13). Moreover, evidence from systematic reviews and meta-analyses also indicate that higher surgeon volume is associated with lower postoperative morbidity and mortality, especially in high-risk operations, such as pancreaticoduodenectomy, colorectal cancer surgery, and gynecology surgery (14-16). Accordingly, considering the advantages from the centralization of high-risk operations, for instance, multidisciplinary expertise and specialized equipment, the trend to centralize them on high-volume surgeons and hospitals is on the way $(17,18)$. Also, there is a potential trend of regionalization in spine surgery, but whether a similar volume-outcomes relationship also existed is unknown and an accumulation of high-grade evidence is lacking (19). Additionally, some studies published recently reported conflicting results (20-30). Two studies suggested that higher volume surgeons were associated with increased postoperative mortality in patients undergoing lumbar spine surgery and anterior cervical fusion $(22,27)$. However, two other studies suggested that there was no significant correlation between surgeon volume and postoperative mortality after lumbar spine surgery and surgery for spinal metastases $(23,26)$.

Therefore, we conducted a dose-response meta-analysis to clarify the relationship between surgeon volume and outcomes in spine surgery, with the hypothesis that higher surgeon volume was associated with lower morbidity and mortality, shorter length of hospital stay, less readmission, and lower hospital costs.

\section{Methods}

The current meta-analysis was performed and reported in accordance with the Meta-analysis of Observational
Studies in Epidemiology (MOOSE) checklist (31). Two reviewers independently undertook the literature selection, data extraction and quality assessment, with inconsistency resolved by the chief reviewer.

\section{Inclusion criteria and search strategy}

We only included observational studies which explored the relationship between surgeon volume and outcomes in spine surgery according to the pre-defined inclusion and exclusion criteria (Table 1). PubMed and Embase were systematically searched without any language limitation from Jan 1946 to Jul 2017. Additionally, apart from PubMed and Embase, The Cochrane Library was added to repetitively search for any eligible studies on Nov 10 2017. Furthermore, an updated search for three databases was conducted for any potential inclusion on Aug 10 2018. The following search terms were used: "volume", "spine surgery" and their variants. In addition, the references of included studies and relevant reviews were carefully checked for any potential inclusion. The updated detailed search strategy is shown in Table S1.

\section{Data extraction and quality assessment}

A standardized Excel table was designed to extract the following information: first author, study period, country, demographic parameter, type of spine surgery, definition of postoperative morbidity, surgeon volume grouping and category (cases/year), study design, covariates in the fully adjusted model, and other outcomes of interest. The primary outcome was postoperative morbidity, which was defined as postoperative complications according to the description of an individual included study (such as any postoperative complications, surgical complications, medical complications). The secondary outcomes consisted of postoperative mortality, length of hospital stay, readmission, and hospital costs. The quality of included studies was assessed using NewcastleOttawa Scale, which including eight domains totaling nine stars, namely exposed cohort, non-exposed cohort, ascertainment of exposure, outcome of interest, comparability, assessment of outcome, length of follow-up, adequacy of follow-up (32). Respectively, 0 to 3, 4 to 6 , and 7 to 9 score were classified as low, moderate, and high quality.

\section{Statistical analysis}

For the binary variable and continuous variable, 
Table 1 Inclusion and exclusion criteria in the current meta-analysis

\begin{tabular}{|c|c|}
\hline Detailed inclusion and exclusion criteria & PICOS principle \\
\hline Intervention/exposure (I) & $\begin{array}{l}\text { High-volume surgeons which were defined as surgeons performing more spine surgeries } \\
\text { per year }\end{array}$ \\
\hline Control (C) & $\begin{array}{l}\text { Low-volume surgeons which were defined as surgeons performing less spine surgeries } \\
\text { per year }\end{array}$ \\
\hline Study design (S) & $\begin{array}{l}\text { - Observational studies with at least two surgeon volume grouping } \\
\text { - Comparative studies without language limitation }\end{array}$ \\
\hline
\end{tabular}

multivariate odds ratios (ORs) with 95\% CIs and weighted mean differences (WMDs) with 95\% CIs between extreme levels of surgeon volume (highest $v s$. lowest) were pooled respectively. Considering substantial clinical heterogeneity among included studies, random-effect model is used to estimate overall pooled effect firstly. If there exist inconsistent results between random and fix-effect model, fix-effect model will be chosen finally, which may reflect the actual effect of higher volume surgeon according to Corhrane Handbook (9.5.4). Statistical heterogeneity across included studies was assessed using $\mathrm{I}^{2}$ statistic and $\mathrm{I}^{2}>50 \%$ was regarded as significant heterogeneity (33). To investigate potential sources of heterogeneity, we performed the "leave-one-out" influence analysis and subgroup analysis for the primary outcome following pre-defined criterion (adult spine surgery $v s$. adolescent spine surgery; lumbar spine surgery $v s$. cervical spine surgery $v s$. spinal tumors surgery; sample size $<100,000$ vs. $>100,000$; surgical complications $v s$. major medical complications vs. any postoperative complications; surgeon volume grouping; high study quality $v s$. moderate or low study quality). Considering that hospital volume, teaching status and operative complexity index may largely introduce bias, we also performed subgroup analyses based on whether these confounders were adjusted or not. Two studies involving anterior cervical fusion were from the
Nationwide Inpatient Sample database and it was difficult to judge whether the study by De la Garza Ramos et al. was included in the study by Basques and colleagues $(20,22)$. Accordingly, we conducted sensitivity analysis through removing the study by De la Garza Ramos and colleagues, which may introduce potential bias in the current metaanalysis. Meanwhile, we performed secondary meta-analysis for the primary outcome through only including unadjusted data. Additionally, we explored the Dose-response relationship of surgeon volume and postoperative morbidity using the method proposed by Greenland et al. $(34,35)$. According to the requirement of the Dose-response analysis method, we only included the studies which provided the following information: the amount of surgeon case volume, distributions of cases, adjusted ORs with $95 \%$ CIs, and at least three surgeon volume grouping. We did not perform the aforementioned analyses for secondary outcomes owing to limited data. if more than 10 studies were included in the current meta-analysis, we evaluated potential publication bias by inspecting funnel plots qualitatively and Begg and Egger tests quantitatively $(36,37)$. If publication bias was detected in the current meta-analysis, the "trim and fill method" was employed to estimate the influence of "missing studies" on overall pooled effect $(38,39)$. All the above statistical analyses were conducted using Review Manager 


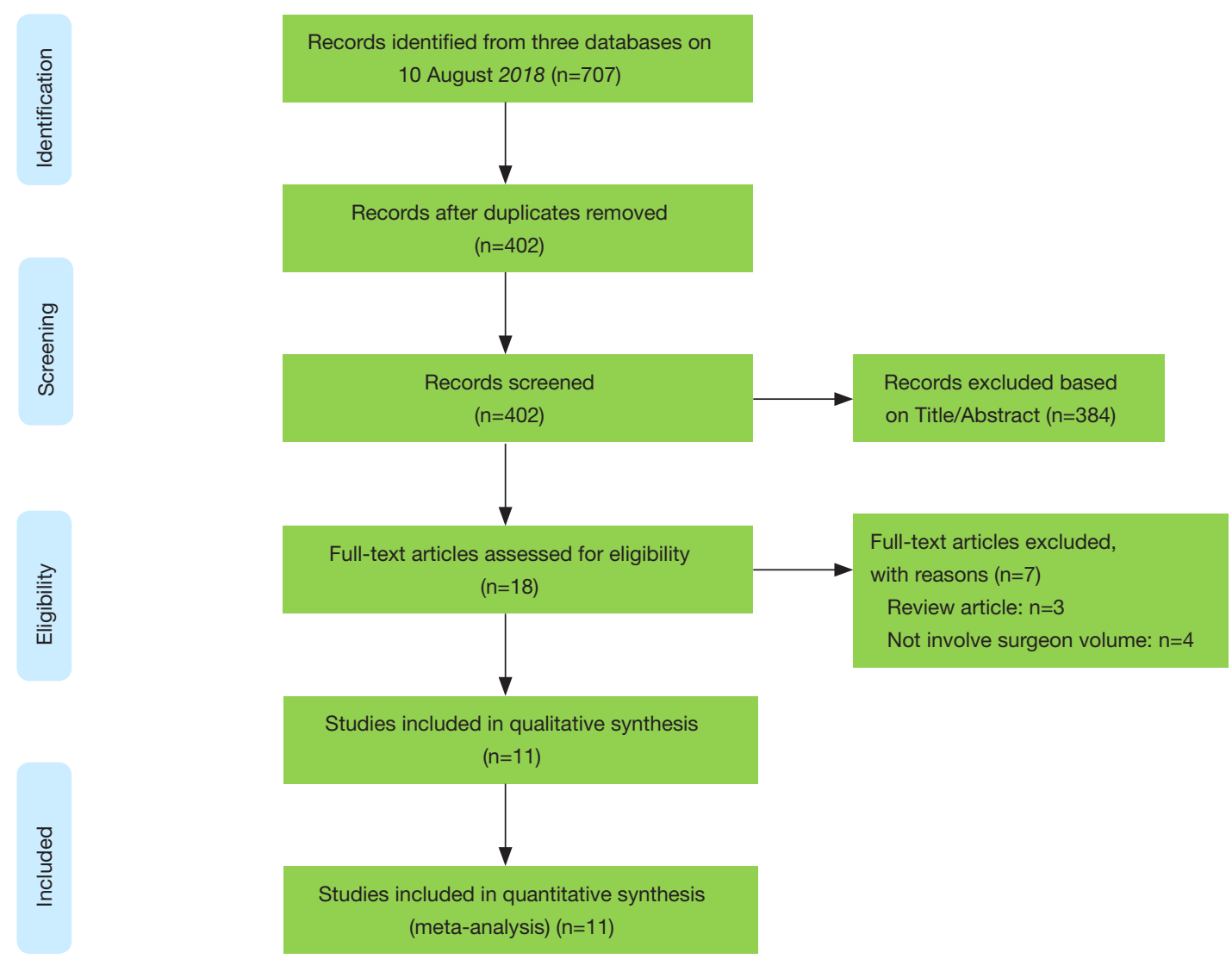

Figure 1 PRISMA flow diagram of literature selection. Adopted form Moher et al. (40).

5.3 and Stata 12.0.

\section{Results}

\section{Study selection}

We obtained 707 items after systematically searching three databases on Aug 102018 (Figure 1). After removing duplicates and irrelevant records, the remaining eighteen studies were further checked for full-text screening. Of these, eleven conformed to the inclusion criteria and none of the additional studies were eligible after carefully checking the references of included studies and important reviews. Finally, eleven studies were included in the current meta-analysis (20-30).

\section{Study characteristics}

Table 2 shows the characteristics of included studies in detail. All the studies were performed in America. The data of 7 studies were from the Nationwide Inpatient Sample
Database $(20,22-27)$ three from Florida Statewide Inpatient Database (28-30) and one from MarketScan Longitudinal Database (21). Three studies involved lumbar spine surgery (26-28), four involved cervical spine surgery (20-22,30), and the others involved spinal deformity surgery or tumor surgery $(23-25,29)$. All the included studies involved adult spine surgery, except the study by Paul and colleagues (24). The definition of postoperative morbidity, surgeon volume grouping and category, and covariates in adjusted mode were also different from each included study. As a whole, the quality of eight studies was classified as high quality $(20,21,24,26-30)$, and three was classified as moderate quality $(22,23,25)$. The detailed NOS score of each individual included study is presented in Table 3.

\section{Postoperative morbidity}

Eleven studies reported relevant data on postoperative morbidity (20-30). Pooled estimates indicated that higher volume surgeon was associated with lower incidence 


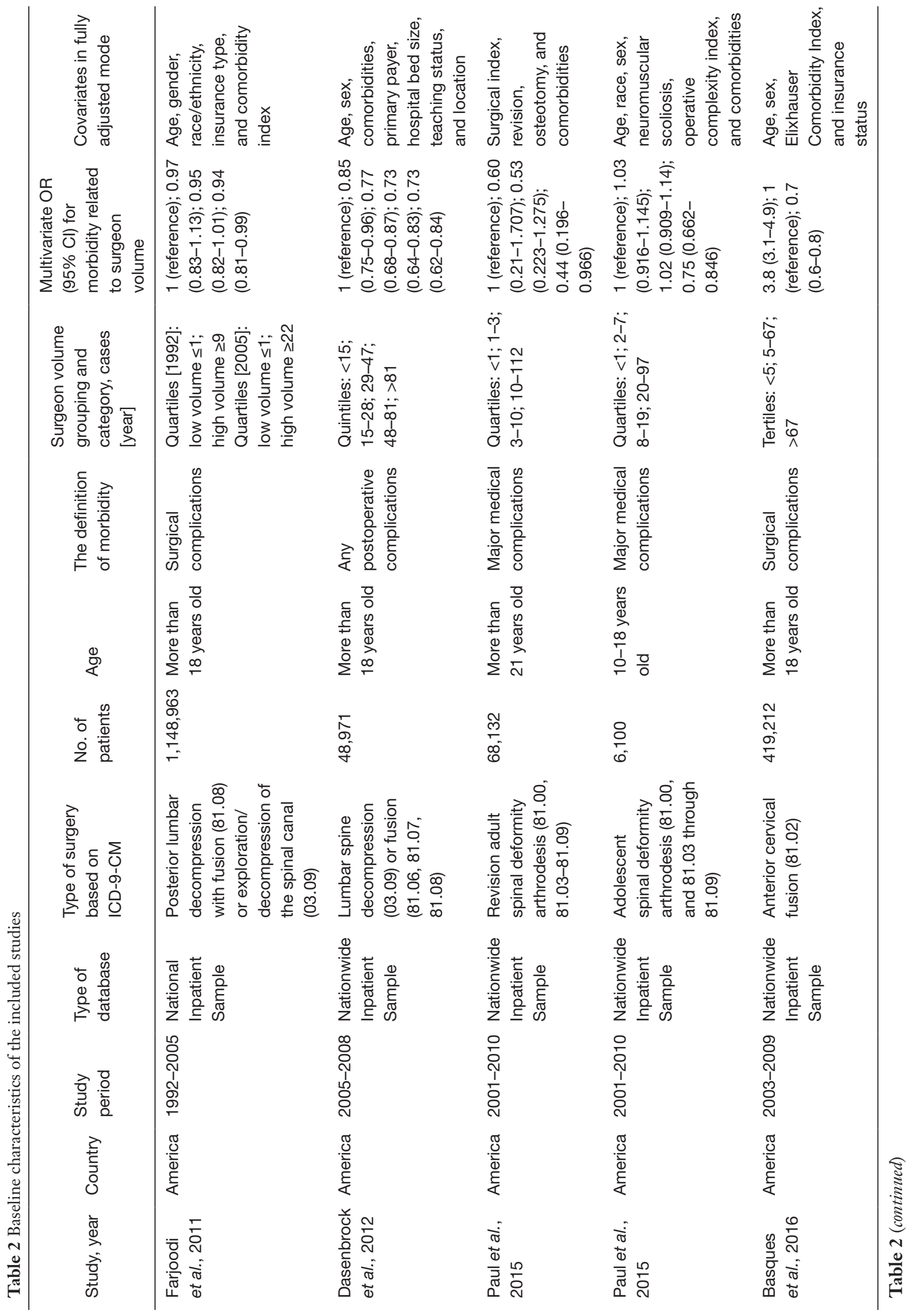




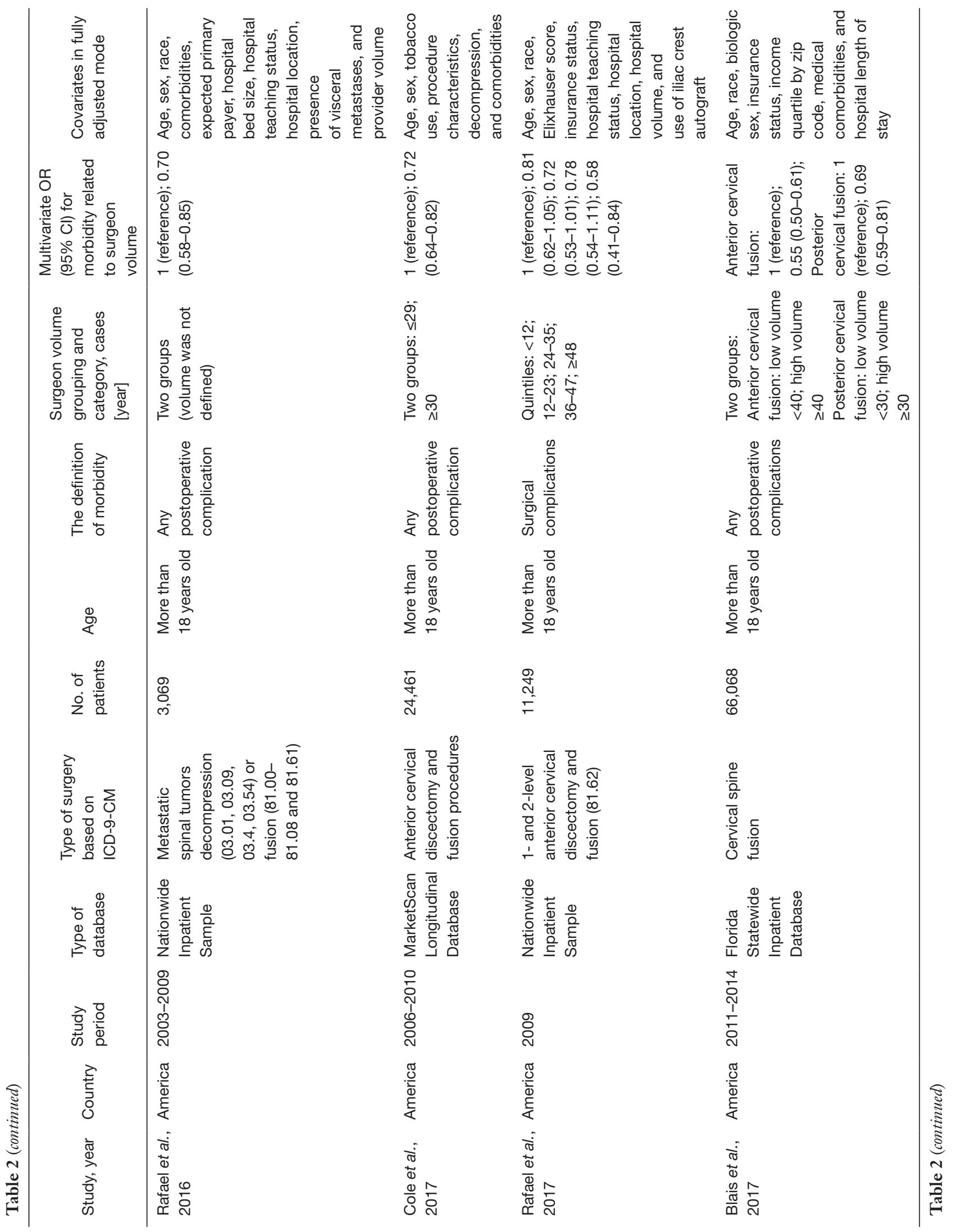




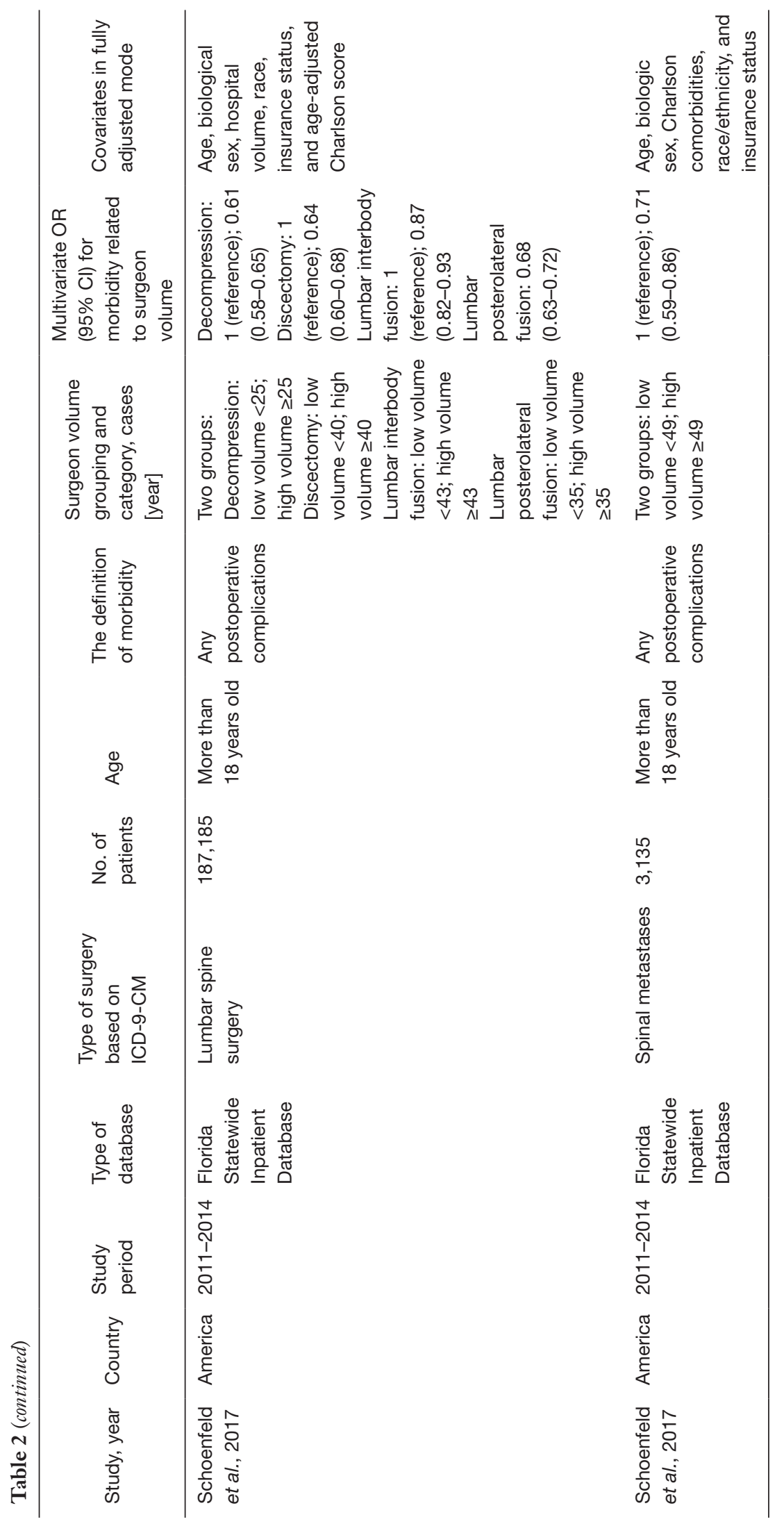


Table 3 Quality assessment using Newcastle-Ottawa Scale

\begin{tabular}{|c|c|c|c|c|c|c|c|c|c|}
\hline \multirow[b]{2}{*}{ Study, years } & \multicolumn{4}{|c|}{ Selection } & \multirow[b]{2}{*}{ Comparability } & \multicolumn{3}{|c|}{ Outcome } & \multirow[b]{2}{*}{ Total score } \\
\hline & $\begin{array}{c}\text { Exposed } \\
\text { cohort }\end{array}$ & $\begin{array}{c}\text { Nonexposed } \\
\text { cohort }\end{array}$ & $\begin{array}{l}\text { Ascertainment } \\
\text { of exposure }\end{array}$ & $\begin{array}{l}\text { Outcome of } \\
\text { interest }\end{array}$ & & $\begin{array}{l}\text { Assessment } \\
\text { of outcome }\end{array}$ & $\begin{array}{l}\text { Length of } \\
\text { follow-up }\end{array}$ & $\begin{array}{l}\text { Adequacy } \\
\text { of follow-up }\end{array}$ & \\
\hline $\begin{array}{l}\text { Dasenbrock } \\
\text { et al., } 2012\end{array}$ & * & * & * & * & ** & * & - & - & 7 \\
\hline Paul et al., 2015 & * & * & * & * & ** & * & - & - & 7 \\
\hline $\begin{array}{l}\text { Basques } \\
\text { et al., } 2016\end{array}$ & * & * & * & * & * & * & - & - & 6 \\
\hline Rafael et al., 2016 & * & * & - & * & ** & * & - & - & 6 \\
\hline Blais et al., 2017 & * & * & * & * & $\star *$ & * & - & - & 7 \\
\hline $\begin{array}{l}\text { Schoenfeld } \\
\text { et al., } 2017\end{array}$ & * & * & * & * & ** & * & - & - & 7 \\
\hline $\begin{array}{l}\text { Schoenfeld } \\
\text { et al., } 2017\end{array}$ & * & * & * & * & ** & * & - & - & 7 \\
\hline
\end{tabular}

*, 1 score; ${ }^{\star \star}, 2$ scores.

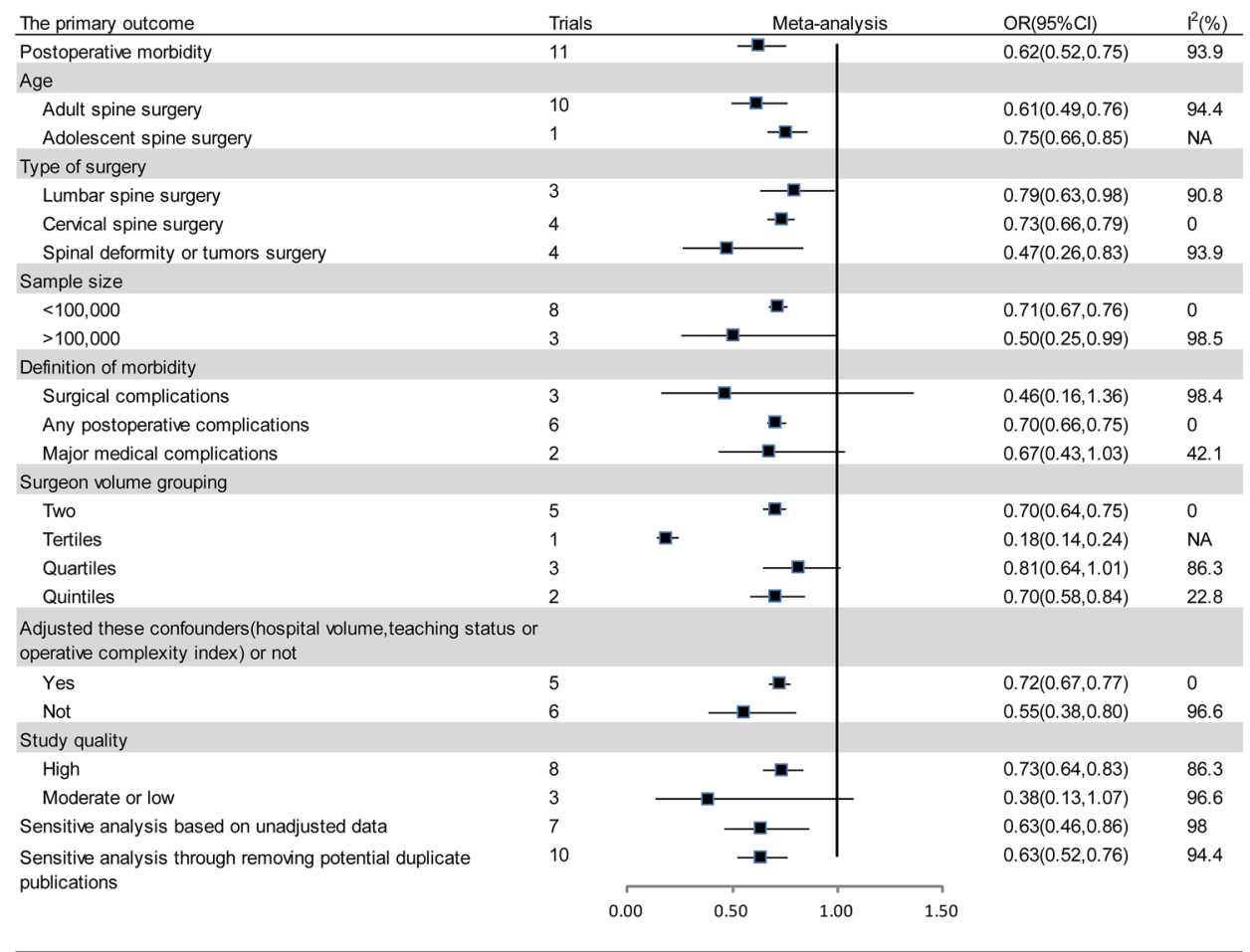

Figure 2 Forest plot summary of the relationship between surgeon volume and postoperative morbidity in subgroup analyses and sensitive analyses. CI, confidence interval; OR, odds ratio. 
of morbidity (OR: $0.62 ; 95 \%$ CI: $0.52-0.75 ; \mathrm{I}^{2}=93.9 \%$; Figure 2). The "leave-one-out" influence analysis suggested that the combined OR basically kept balance from 0.60 (95\% CI: 0.49-0.72) to 0.72 (95\% CI: 0.63-0.81). Also, to explore the possible sources of statistical heterogeneity, we performed subgroup analysis and sensitive analysis (pooling unadjusted data or removing potential duplicate publications). Pooled results of above additional analyses are basically consistent with the overall pooled estimate (Figure 2). We also conducted a secondary doseresponse meta-analysis for the primary outcome and five studies were included in the dose-response analysis $(20,22,24-26)$. We found a nonlinear Dose-response relationship between surgeon volume and postoperative morbidity ( $\mathrm{P}$ for nonlinearity less than 0.00001 ). The non-linearity relationship between surgeon volume and postoperative morbidity indicated that increased surgeon volume was accompanied with the decline of postoperative morbidity (Figure 3).

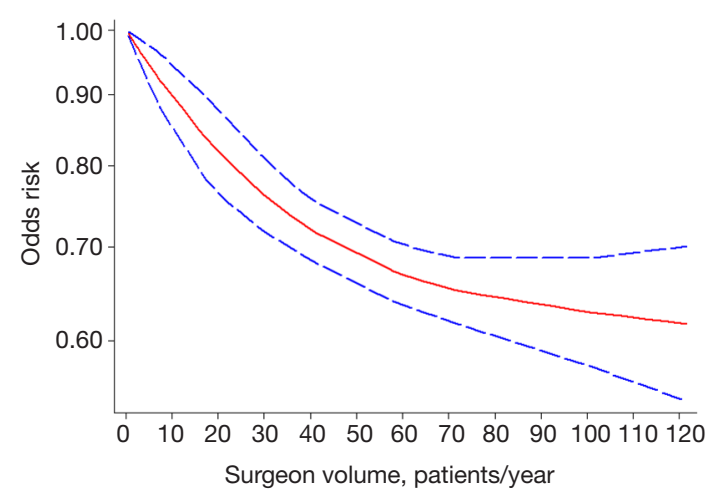

Figure 3 Dose-response analysis of the relationship between surgeon volume and postoperative morbidity in spine surgery.

\section{The secondary outcomes}

The pooled effect of length of hospital stay and hospital costs are inconsistent with substantial heterogeneity after using random and fix-effect model. Therefore, the results of fix-effect model were chosen finally, which may reflect the actual effect of higher volume surgeon. Pooled analysis indicated that higher surgeon volume was associated with lower incidence of postoperative mortality ( 4 studies; OR: 0.76; 95\% CI: 0.66-0.87; I I =0; Table 4), shorter length of hospital stay (3 studies; WMD: -7.07 ; 95\% CI: -7.08 to $-7.06 ; \mathrm{I}^{2}=100 \%$; Table 4), less readmission (4 studies; OR, 0.78; 95\% CI: $0.72-0.85 ; \mathrm{I}^{2}=93.1 \%$; Table 4$)$ and lower hospital costs (2 studies; WMD: $-25,497.47 ; 95 \%$ CI: $-25,528.43$ to $-25,466.51 ; \mathrm{I}^{2}=100 \%$; Table 4).

\section{Publication bias}

The funnel plot seemed to be visually asymmetrical, which was subsequently verified by statistical test $(\mathrm{Begg}, \mathrm{P}=0.062$; Egger, $\mathrm{P}=0.005)$. Considering that there existed potential publication bias, we further conducted the "trim and fill" method. The result showed that two "missing studies" with negative results may be the underlying source of publication bias. Nevertheless, the overall pooled effect kept balanced after adding these "missing studies" in the current metaanalysis (13 studies; OR, 0.57; 95\% CI: 0.43-0.75, Figure 4).

\section{Discussion}

\section{Key findings}

Our study indicated that higher surgeon volume was associated with lower postoperative morbidity and mortality, shorter length of hospital stay, less readmission and lower hospital costs in spine surgery. Additionally, there existed

Table 4 Meta-analyses for secondary outcomes

\begin{tabular}{lcccc}
\hline Outcomes & Trials & ES $(95 \%$ Cl)* & P for overall effect & $I^{2}(\%)$ \\
\hline Postoperative mortality & 4 & OR: $0.76(0.66,0.87)$ & $<0.00001$ & 0 \\
Length of hospital stay & 3 & WMD: $-7.07(-7.08,-7.06)$ & $<0.00001$ & 100 \\
Readmission & 4 & OR: $0.78(0.72,0.85)$ & $<0.00001$ & 93.1 \\
Hospital costs & 2 & WMD: $-25,497.47(-25,528.43,-25,466.51)$ & $<0.00001$ & 100 \\
\hline
\end{tabular}

*, ORs with $95 \% \mathrm{Cl}$ was estimated using mantel-haenszel statistical method and random-effects model, and WMDs with $95 \% \mathrm{Cl}$ was estimated using inverse variance statistical method and fix-effects model. OR, odds ratio; WMD, weighted mean difference; ES, effect size. 


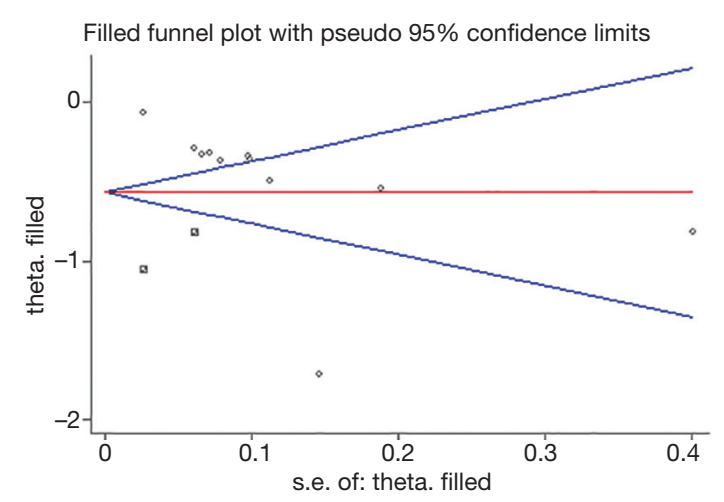

Figure 4 Funnel plot of the surgeon volume-outcomes in spine surgery. Statistic test (Begg, $\mathrm{P}=0.062$; Egger, $\mathrm{P}=0.005$ ) suggested potential publication. And then, 'trim and fill' method suggested that two 'missing studies' with negative results may be the possible source of publication bias. However, the overall pooled effect estimate remained steady when these 'missing studies' were added in the current meta-analysis (13 studies; OR, 0.57; 95\% CI: 0.43-0.75). Circle: eleven included studies. Square: two "missing studies" from the estimation of trim and fill method. SE, standard error.

a non-linear Dose-response relationship between surgeon volume and postoperative morbidity in spine surgery.

\section{Interpretation of main results}

The inverse volume-outcomes relationship was demonstrated in spine surgeries. Actually, similar surgeon volume-outcomes relationship has been verified in shoulder arthroplasty, which suggested that low surgeon volume (i.e., those performing $<5$ arthroplasties per year) was associated with increased surgical complications, length of hospital stay, hospital time, and hospital costs (41). The current study was based on 11 studies with substantial heterogeneity $\left(\mathrm{I}^{2}=93.9 \%\right)$. The sources of statistical heterogeneity weren't identified through subsequent subgroup analyses and sensitivity analyses, which may weaken the interpretation of the pooled effect. Understandably, there were several clinical and methodological differences across included studies, such as demographic characteristics, type of spine surgery, the definition of postoperative morbidity, the cutoff value of surgeon volume grouping and study design. Thus, the sources of statistical heterogeneity were inherent and multi-factorial and individual risk factor cannot be attributed to it. Anyhow, the results of subgroup analysis and sensitivity analyses were basically consistent with the overall pooled effect, which at least suggesting that the current available evidence was robust and reliable. Meanwhile, the dose-response analysis also indicated that the higher surgeon case volume was accompanied with lower morbidity in spine surgery, although the inverse relationship was non-linear.

\section{Potential mechanism and clinical application}

Our investigation indicated that higher surgeon volume was associated with lower morbidity and mortality. Two potential mechanism may explain the "volume-outcome" relationship in our study. The first one is "practicemakes-perfect', that is, high volume surgeons with superb surgical skills and much surgical experience can translate into better prognosis and this explanation means that high surgeon volume contribute to high-quality medical supply (42). Actually, the mechanism is also suitable for other complicated high-risk surgeries, such as esophagectomy, gastrectomy, and pancreatectomy (43), but not low-risk surgeries, such as cholecystectomy (44). Meanwhile, high-volume surgeons in high-volume hospitals usually possess better specialization and their specialized training could translate into better prognosis in complicated operations. A recent study suggested that increased surgeon specialization was associated with lower rates of postoperative morbidity and mortality in spine surgery (45). The second potential mechanism is 'selective-referral patterns', that is, patients with complex surgical conditions tend to go to high-volume hospitals and these hospitals usually possess multidisciplinary care teams and experienced surgeons. The explanation means that high-quality medical supply contribute to high surgeon volume (46).

Given better prognosis from the treatment of higher volume surgeons, the conclusions of our study may provide some potential clinical implications for clinical policy makers and further clinical studies. On one hand, the volume-outcomes relationship in spine surgery suggest that centralization of spine surgery in high-volume surgical providers can maximize patients' benefit. A 10-year retrospective analysis suggested that patients with lumbar spine diseases tended to go to high-volume hospitals for operation, irrespective of policy makers' efforts aimed at encouraging patients to high-volume hospitals $(19,47)$. As the market share of high-volume hospitals increases, what we can forecast is that more patient populations will gather in high-level hospitals and the disparities of spine surgeries among high- and low-volume hospitals will be 
further enlarged in the foreseeable future. More alarmingly, the overload of patients waiting for operation in highvolume hospitals may induce the relative shortage of medical resources, such as high-volume surgeons, nurses, beds, and other surgical equipment. Conversely, lowvolume hospitals will have many idle low-volume surgeons and nurses, and unused beds and surgical equipment. Therefore, clinical policy makers should focus on the balance of medical resources among high- and low-volume hospitals when considering their potential relative shortage in high-volume hospitals. One the other hand, regardless of the advantages of centralization of spine surgery, there were some potential barriers to implement it (48). For instance, patients were willing to go to hospitals close to their homes and high-volume hospitals with high-volume surgeons were usually far from their homes. Recent studies also indicated that minorities, such as African Americans or Hispanics, and patients with Medicaid had difficulty in accessibility to operation at high-volume hospitals (49). Thus, race and socio-economic status may be responsible for the inaccessibility of urban patients to the high-quality treatment. Accordingly, further clinical policy should also center on removing these potential barriers in the process of centralization of spine surgery in these vulnerable populations.

\section{Strengths and limitations}

A main strength of the current study lies in its accordance with MOOSE checklist. We collected any possible eligible studies through exhaustive search according to predefined inclusion and exclusion criteria. Two reviewers independently conducted the literature search and selection, data extraction and quality assessment to make sure the processes repeatable and reproducible. In addition, a dose-response meta-analysis was undertaken to assess the reliability of the present available evidence.

Our study also had some limitations. Firstly, we basically confirmed the inverse volume-outcomes relationship in spine surgery, but the results should be interpreted with caution owing to the heterogeneous definition of high-and low-volume surgeons across included studies. Accordingly, further studies focusing on more homogeneous definition of low- and high-volume surgeon are warranted. Moreover, the consistency may have potential implication for formulating the best caseload standard of spinal surgeons for clinical policy makers. Secondly, the statistical heterogeneity was significant $\left(\mathrm{I}^{2}=93.9 \%\right)$ and the sources of statistical heterogeneity weren't identified through subsequent subgroup analyses and sensitivity analyses, so further studies with relative homogeneous clinical scenario should be warranted. Thirdly, the current investigation was merely based on eleven observational studies and the whole quality of included studies was moderate. Also, observational studies usually introduced potential confounding bias and selection bias, although we merely pooled adjusted OR in full adjusted mode. Fourthly, all the included studies were conducted in America, so the conclusions had limited applicability in other regions, such as Asia and Europe. Fifthly, pooled estimate based on fix-effect model only indicated that high volume surgeon was better than low volume surgeon on continuous outcomes. However, the authentic quantitative effect of high volume surgeon on length of hospital stay and hospital costs was largely unclear owing to limited available studies and substantial clinical heterogeneity. Finally, our study existed potential publication bias, although we performed a comprehensive literature search. However, the overall pooled effect kept balanced after adding these "missing studies". These limitations may impair the interpretation of our main results and the conclusions should be interpreted with caution. Further high-quality studies should be warranted to clarify the relationship between surgeon volume and outcomes in spine surgery.

\section{Conclusions}

The current evidence indicated that higher surgeon volume was associated with lower morbidity and mortality, shorter length of hospital stay, less readmission and lower hospital costs. Further studies should be warranted to define the optimal volume thresholds of spine surgeries for surgeons, which will contribute to improve their clinical skill and maximize patients' benefit.

\section{Acknowledgements}

The authors thank Liu Zhong (Department of Neurosurgery, the First Affiliated Hospital of Sun Yatsen University, Guangzhou, China) for his substantial contribution to editing the funnel plot.

Funding: This study was supported by grants from the National Natural Science Foundation of China (No. 81772384; 81572174). The funders had no role in study design, data collection and analysis, decision to publish, or preparation of the manuscript. 


\section{Footnote}

Conflicts of Interest: The authors have no conflicts of interest to declare.

\section{References}

1. Bernstein DN, Brodell D, Li Y, et al. Impact of the Economic Downturn on Elective Lumbar Spine Surgery in the United States: A National Trend Analysis, 2003 to 2013. Global Spine J 2017;7:213-9.

2. Bernstein DN, Jain A, Brodell D, et al. Impact of the Economic Downturn on Elective Cervical Spine Surgery in the United States: A National Trend Analysis, 20032013. World Neurosurg 2016;96:538-44.

3. Zygourakis CC, Liu CY, Keefe M, et al. Analysis of National Rates, Cost, and Sources of Cost Variation in Adult Spinal Deformity. Neurosurgery 2018;82:378-87.

4. Saleh A, Thirukumaran C, Mesfin A, et al. Complications and readmission after lumbar spine surgery in elderly patients: an analysis of 2,320 patients. Spine J 2017;17:1106-12.

5. Baker JF, McClelland S, 3rd, Line BG, et al. In-hospital Complications Following Lumbar Spine Surgery in Patients with Parkinson's Disease: Evaluation of the National Inpatient Sample Database. World Neurosurg 2017;106:470-6.

6. Novais EN, Carry PM, Kestel LA, et al. Does Surgeon Experience Impact the Risk of Complications After Bernese Periacetabular Osteotomy? Clin Orthop Relat Res 2017;475:1110-7.

7. Ahn J, Iqbal A, Manning BT, et al. Minimally invasive lumbar decompression-the surgical learning curve. Spine J 2016;16:909-16.

8. Ravi B, Croxford R, Austin PC, et al. Increased surgeon experience with rheumatoid arthritis reduces the risk of complications following total joint arthroplasty. Arthritis Rheumatol 2014;66:488-96.

9. Maruthappu M, Gilbert BJ, El-Harasis MA, et al. The influence of volume and experience on individual surgical performance: a systematic review. Ann Surg 2015;261:642-7.

10. Hannan EL, O'Donnell JF, Kilburn H Jr, et al. Investigation of the relationship between volume and mortality for surgical procedures performed in New York State hospitals. JAMA 1989;262:503-10.

11. Markar SR, Mackenzie H, Askari A, et al. Effect of Esophageal Cancer Surgeon Volume on Management and Mortality From Emergency Upper Gastrointestinal
Conditions: Population-based Cohort Study. Ann Surg 2017;266:847-53.

12. Choi JW, Jeon SW, Kwon JG, et al. Volume of hospital is important for the prognosis of high-risk patients with nonvariceal upper gastrointestinal bleeding (NVUGIB). Surg Endosc 2017;31:3339-46.

13. Waingankar N, Mallin K, Smaldone M, et al. Assessing the relative influence of hospital and surgeon volume on short-term mortality after radical cystectomy. BJU Int 2017;120:239-45.

14. Macedo FIB, Jayanthi P, Mowzoon M, et al. The Impact of Surgeon Volume on Outcomes After Pancreaticoduodenectomy: a Meta-analysis. J Gastrointest Surg 2017.

15. Huo YR, Phan K, Morris DL, et al. Systematic review and a meta-analysis of hospital and surgeon volume/outcome relationships in colorectal cancer surgery. J Gastrointest Oncol 2017;8:534-46.

16. Mowat A, Maher C, Ballard E. Surgical outcomes for lowvolume vs high-volume surgeons in gynecology surgery: a systematic review and meta-analysis. Am J Obstet Gynecol 2016;215:21-33.

17. Laucis NC, Chowdhury M, Dasgupta A, et al. Trend Toward High-Volume Hospitals and the Influence on Complications in Knee and Hip Arthroplasty. J Bone Joint Surg Am 2016;98:707-12.

18. Gani F, Azoulay D, Pawlik TM. Evaluating Trends in the Volume-Outcomes Relationship Following Liver Surgery: Does Regionalization Benefit All Patients the Same? J Gastrointest Surg 2017;21:463-71.

19. Jancuska J, Adrados M, Hutzler L, et al. The Regionalization of Lumbar Spine Procedures in New York State: A 10-Year Analysis. Spine (Phila Pa 1976) 2016;41:153-8.

20. De la Garza Ramos R, Nakhla J, Nasser R, et al. Volumeoutcome relationship after 1 and 2 level anterior cervical discectomy and fusion. World Neurosurg 2017;105:543-8.

21. Cole T, Veeravagu A, Zhang M, et al. Surgeon Procedure Volume and Complication Rates in Anterior Cervical Discectomy and Fusions: Analysis of a National Longitudinal Database. Clin Spine Surg 2017;30:E633-9.

22. Basques BA, Louie PK, Shifflett GD, et al. Effect of Surgeon Volume on Complications, Length of Stay, and Costs Following Anterior Cervical Fusion. Spine (Phila Pa 1976) 2017;42:394-9.

23. De la Garza-Ramos R, Abt NB, Kerezoudis P, et al. Provider volume and short-term outcomes following surgery for spinal metastases. J Clin Neurosci 2016;24:43-6. 
24. Paul JC, Lonner BS, Toombs CS. Greater operative volume is associated with lower complication rates in adolescent spinal deformity surgery. Spine (Phila Pa 1976) 2015;40:162-70.

25. Paul JC, Lonner BS, Goz V, et al. Complication rates are reduced for revision adult spine deformity surgery among high-volume hospitals and surgeons. Spine J 2015;15:1963-72.

26. Dasenbrock HH, Clarke MJ, Witham TF, et al. The impact of provider volume on the outcomes after surgery for lumbar spinal stenosis. Neurosurgery 2012;70:1346-53; discussion 1353-4.

27. Farjoodi P, Skolasky RL, Riley LH. The effects of hospital and surgeon volume on postoperative complications after lumbar spine surgery. Spine 2011;36:2069-75.

28. Schoenfeld AJ, Sturgeon DJ, Burns CB, et al. Establishing benchmarks for the volume-outcome relationship for common lumbar spine surgical procedures. Spine J 2018;18:22-8.

29. Schoenfeld AJ, Ferrone ML, Sturgeon DJ, et al. VolumeOutcome Relationship in Surgical Interventions for Spinal Metastases. J Bone Joint Surg Am 2017;99:1753-9.

30. Blais MB, Rider SM, Sturgeon DJ, et al. Establishing objective volume-outcome measures for anterior and posterior cervical spine fusion. Clin Neurol Neurosurg 2017;161:65-9.

31. Stroup DF, Berlin JA, Morton SC, et al. Meta-analysis of observational studies in epidemiology: a proposal for reporting. Meta-analysis Of Observational Studies in Epidemiology (MOOSE) group. JAMA 2000;283:2008-12.

32. Kim SY, Park JE, Lee YJ, et al. Testing a tool for assessing the risk of bias for nonrandomized studies showed moderate reliability and promising validity. J Clin Epidemiol 2013;66:408-14.

33. Higgins JP, Thompson SG, Deeks JJ, et al. Measuring inconsistency in meta-analyses. BMJ 2003;327:557-60.

34. Greenland S, Longnecker MP. Methods for trend estimation from summarized dose-response data, with applications to meta-analysis. Am J Epidemiol 1992;135:1301-9.

35. Orsini N, Li R, Wolk A, et al. Meta-analysis for linear and nonlinear dose-response relations: examples, an evaluation of approximations, and software. Am J Epidemiol 2012;175:66-73.

36. Begg CB, Mazumdar M. Operating characteristics of a rank correlation test for publication bias. Biometrics 1994;50:1088-101.

37. Egger M, Davey Smith G, Schneider M, et al. Bias in meta-analysis detected by a simple, graphical test. BMJ
1997;315:629-34.

38. Duval S, Tweedie R. Trim and fill: A simple funnel-plotbased method of testing and adjusting for publication bias in meta-analysis. Biometrics 2000;56:455-63.

39. Peters JL, Sutton AJ, Jones DR, et al. Performance of the trim and fill method in the presence of publication bias and between-study heterogeneity. Stat Med 2007;26:4544-62.

40. Moher D, Liberati A, Tetzlaff J, et al. Preferred reporting items for systematic reviews and meta-analyses: the PRISMA statement. PLoS Med 2009;6:e1000097

41. Weinheimer KT, Smuin DM, Dhawan A. Patient Outcomes as a Function of Shoulder Surgeon Volume: A Systematic Review. Arthroscopy 2017;33:1273-81.

42. Luft HS, Hunt SS, Maerki SC. The volume-outcome relationship: practice-makes-perfect or selective-referral patterns? Health Serv Res 1987;22:157-82.

43. Mamidanna R, Ni Z, Anderson O, et al. Surgeon Volume and Cancer Esophagectomy, Gastrectomy, and Pancreatectomy: A Population-based Study in England. Ann Surg 2016;263:727-32.

44. Abelson JS, Spiegel JD, Afaneh C, et al. Evaluating cumulative and annual surgeon volume in laparoscopic cholecystectomy. Surgery 2017;161:611-7.

45. McCutcheon BA, Hirshman BR, Gabel BC, et al. Impact of neurosurgeon specialization on patient outcomes for intracranial and spinal surgery: a retrospective analysis of the Nationwide Inpatient Sample 1998-2009. J Neurosurg 2018;128:1578-88.

46. Dudley RA, Johansen KL, Brand R, et al. Selective referral to high-volume hospitals: estimating potentially avoidable deaths. JAMA 2000;283:1159-66.

47. Jancuska JM, Hutzler L, Protopsaltis TS, et al. Utilization of Lumbar Spinal Fusion in New York State: Trends and Disparities. Spine (Phila Pa 1976) 2016;41:1508-14.

48. Chhabra KR, Dimick JB. Strategies for Improving Surgical Care: When Is Regionalization the Right Choice? JAMA Surg 2016;151:1001-2.

49. Feng R, Finkelstein M, Bilal K, et al. Trends and Disparities in Cervical Spine Fusion Procedures Utilization in the New York State. Spine (Phila Pa 1976) 2018;43:E601-6.

Cite this article as: Li HZ, Lin Z, Li ZZ, Yang ZY, Zheng Y, Li Y, Lu HD. Relationship between surgeon volume and outcomes in spine surgery: a dose-response meta-analysis. Ann Transl Med 2018;6(22):441. doi: 10.21037/atm.2018.10.48 
Table S1 Detailed search strategy in PubMed and Embase, and The Cochrane Library

\begin{tabular}{|c|c|c|}
\hline Search & Query & Items found \\
\hline \multicolumn{3}{|c|}{ Source: PubMed; searched on: Aug 10, 2018; results: 266} \\
\hline$\# 1$ & Search spine[Title] & 33,254 \\
\hline \#2 & Search spinal[Title] & 116,580 \\
\hline \#3 & Search vertebrae[Title] & 2,931 \\
\hline \#4 & Search vertebral[Title] & 19,775 \\
\hline \#5 & Search cervical[Title] & 96,827 \\
\hline \#6 & Search Lumbar[Title] & 36,165 \\
\hline \#7 & Search thoracic[Title] & 45,623 \\
\hline \#8 & Search sacral[Title] & 5,639 \\
\hline$\# 9$ & Search pedicle[Title] & 6,641 \\
\hline$\# 10$ & Search (\#1 OR \#2 OR \#3 OR \#4 OR \#5 OR \#6 OR \#7 OR \#8 OR \#9) & 323,321 \\
\hline$\# 11$ & Search "hospital volumes" [Title/Abstract] & 133 \\
\hline$\# 12$ & Search "surgeon volumes" [Title/Abstract] & 75 \\
\hline$\# 13$ & Search "provider volumes" [Title/Abstract] & 25 \\
\hline$\# 14$ & Search "hospital volume"[Title/Abstract] & 1,565 \\
\hline$\# 15$ & Search "surgeon volume"[Title/Abstract] & 753 \\
\hline$\# 16$ & Search "provider volume"[Title/Abstract] & 137 \\
\hline$\# 17$ & Search "regionalization"[Title/Abstract] & 2,605 \\
\hline$\# 18$ & Search "regionalisation"[Title/Abstract] & 266 \\
\hline$\# 19$ & Search "Workload"[Title/Abstract] & 21,197 \\
\hline$\# 20$ & Search ((\#11 OR \#12 OR \#13 OR \#14 OR \#15 OR \#16 OR \#17 OR \#18 OR \#19)) & 26,142 \\
\hline \#21 & Search (\#10 AND \#20) & 266 \\
\hline \multicolumn{3}{|c|}{ Source: Embase; searched on: Aug 10, 2018; results: 370} \\
\hline$\# 1$ & spine:ti & 40,879 \\
\hline$\# 2$ & vertebrae:ti & 3,115 \\
\hline \#3 & vertebral:ti & 25,124 \\
\hline$\# 4$ & spinal:ti & 139,404 \\
\hline \#5 & cervical:ti & 120,270 \\
\hline \#6 & lumbar:ti & 44,142 \\
\hline$\# 7$ & thoracic:ti & 54,830 \\
\hline \#8 & sacral:ti & 7,489 \\
\hline$\# 9$ & pedicle:ti & 7,811 \\
\hline$\# 10$ & \#1 OR \#2 OR \#3 OR \#4 OR \#5 OR \#6 OR \#7 OR \#8 OR \#9 & 394,810 \\
\hline$\# 11$ & "hospital volumes":ab,ti & 199 \\
\hline$\# 12$ & "surgeon volumes":ab,ti & 107 \\
\hline$\# 13$ & "provider volumes":ab,ti & 29 \\
\hline$\# 14$ & "hospital volume":ab,ti & 2,238 \\
\hline$\# 15$ & "surgeon volume":ab,ti & 1,050 \\
\hline$\# 16$ & "provider volume":ab,ti & 184 \\
\hline$\# 17$ & "regionalization":ab,ti & 3,092 \\
\hline$\# 18$ & "regionalisation":ab,ti & 337 \\
\hline$\# 19$ & “Workload”: ab,ti & 28,784 \\
\hline$\# 20$ & \#11 OR \#12 OR \#13 OR \#14 OR \#15 OR \#16 OR \#17 OR \#18 OR \#19 & 35,227 \\
\hline \#21 & \#10 AND \#20 & 370 \\
\hline \multicolumn{3}{|c|}{ Source: The Cochrane Library; searched on: Aug 10, 2018; results: 71} \\
\hline$\# 1$ & spine:ti,ab,kw & 11,001 \\
\hline$\# 2$ & vertebrae:ti,ab,kw & 4,866 \\
\hline$\# 3$ & vertebral:ti,ab,kw & 3,320 \\
\hline$\# 4$ & spinal:ti,ab,kw & 19,384 \\
\hline \#5 & cervical:ti,ab,kw & 13,736 \\
\hline \#6 & lumbar:ti,ab,kw & 11,616 \\
\hline$\# 7$ & thoracic:ti,ab,kw & 10,442 \\
\hline \#8 & sacral:ti,ab,kw & 896 \\
\hline$\# 9$ & pedicle:ti,ab,kw & 841 \\
\hline$\# 10$ & \#1 OR \#2 OR \#3 OR \#4 OR \#5 OR \#6 OR \#7 OR \#8 OR \#9 & 52,195 \\
\hline$\# 11$ & "hospital volumes":ti,ab,kw & 2 \\
\hline$\# 12$ & "surgeon volumes":ti,ab,kw & 2 \\
\hline$\# 13$ & “provider volumes":ti,ab,kw & 1 \\
\hline$\# 14$ & "hospital volume”:ti,ab,kw & 59 \\
\hline$\# 15$ & "surgeon volume":ti,ab,kw & 32 \\
\hline$\# 16$ & "provider volume":ti,ab, kw & 3 \\
\hline$\# 17$ & "regionalization":ti,ab,kw & 25 \\
\hline$\# 18$ & "regionalisation":ti,ab,kw & 25 \\
\hline$\# 19$ & "Workload":ti,ab,kw & 2,705 \\
\hline$\# 20$ & \#11 OR \#12 OR \#13 OR \#14 OR \#15 OR \#16 OR \#17 OR \#18 OR \#19 & 2,807 \\
\hline \#21 & $\# 10$ AND \#20 & 71 \\
\hline
\end{tabular}

